\title{
POLÍTICAS DE GÉNERO UNIVERSITARIAS: INSTITUCIONALIZACIÓN, TRANSVERSALIZACIÓN Y PARTICIPACIÓN DE LA COMUNIDAD
}

\author{
UNIVERSITY GENDER POLICIES: INSTITUTIONALIZATION, \\ TRANSVERSALIZATION AND COMMUNITY PARTICIPATION
}

\author{
Pedro Octavio Arce Casas. \\ Universidad de Guadalajara - México \\ pedro.arce@udgvirtual.udg.mx
}

Resumen: Las Instituciones de Educación Superior (IES) mexicanas experimentaron, a partir de la segunda mitad del siglo $X X$, un crecimiento en la matrícula, particularmente en las mujeres. Esto podría indicar cierto grado de equidad de género, al menos, en la población estudiantil. Sin embargo, la creencia de que las universidades están aisladas de las problemáticas sociales de los países en que se ubican, está en tela de juicio. Las cada vez más abundantes denuncias de acoso y hostigamiento sexual dentro de las instituciones, así como las correspondientes reacciones de grupos feministas, muestran lo contrario, dejando al descubierto una vulnerabilidad que exhibe a las universidades como instituciones en que se reproduce la dominación masculina predominante en el contexto nacional. En consecuencia, las autoridades se han visto obligadas a actuar y, mediante esfuerzos aislados, pusieron en marcha políticas con perspectiva de género. Por lo que el objetivo de este trabajo es analizar en el marco de las políticas universitarias de género tres conceptos: la institucionalización, la transversalidad y la participación comunitaria que son requisitos necesarios para lograr instituciones incluyentes y seguras para la mitad de la comunidad estudiantil que constituye la abrumante mayoría de población afectada por la violencia de género.

Palabras clave: políticas universitarias de género, violencia de género, institucionalización, transversalización, participación comunitaria.

Abstract: Mexican Higher Education Institutions (HEI) experienced since the second half of the XX century a growth in its enrolments, especially in the female alumni. This aforementioned phenomenon could show certain advancement in gender equality, at least in students' population. However, the belief that Universities are isolated from the social issues of the countries they are established is dubious. The greater amount of complains about sexual harassment inside the $\mathrm{HEI}$, and the reactions from feminist groups reveal the opposite, they have unveiled a vulnerability exhibited by Universities as institutions that reproduce the male domination predominant in the national context. In 
consequence, $\mathrm{HEI}$ authorities have been forced to act, and by isolated efforts started policies from a gender scope. So, the objective of this paper is to analyze within HEI policies framework three concepts: institutionalization, transversalization and community participation, which are requisites to obtain inclusive and safe institutions for half of the student community that conform the overwhelming majority of the population affected by gender violence.

Keywords: university gender policies, gender violence, institutionalization, transversalization, community participation.

\section{Antecedentes en el contexto universitario mexicano}

En términos demográficos, el fenómeno denominado "Expansión de la Educación Superior" equilibró la proporción de hombres y mujeres que tienen acceso a las Instituciones de la Educación Superior mexicanas (Mercado y Planas, 2005; De Garay y del Valle-Diaz-Muñoz, 2011). No obstante, este fenómeno no se ha visto acompañado de una Política con Perspectiva de Género. De acuerdo con Palomar Verea, esta última consiste en "promover un cambio ético en las instituciones para que éstas incorporen en sus sistemas axiológicos el respeto a la diversidad y la búsqueda de la equidad, la de género en particular" (2005, p. 11). Como se puede observar, el planteamiento implica un cambio en la cultura institucional. En sentido contrario, en la discusión de dicha problemática en el ámbito de las universidades, la académica señala que en 2005 la composición de la matrícula cubre sólo uno de los cuatro niveles en los que se planteaba el cambio institucional. Los otros corresponden a la creación de nuevos campos disciplinares, al institucional y al epistemológico. A continuación, proponemos un acercamiento a los tres niveles mencionados.

Como primer elemento está el análisis a nivel institucional. Un aspecto fundamental en el plano de las políticas públicas y, como lo señalan Barreto Ávila y Flores Garrido (2016), preocupante desde una perspectiva de análisis feminista, es que la universidad está pasos atrás en lo tocante a las políticas de género en comparación con las medidas que ha puesto en vigor el gobierno mexicano. La revisión histórica de la implementación de políticas con perspectiva de equidad de género ha sido en forma de "cascada" (p. 205), es decir, empieza por la puesta en marcha de políticas en la esfera internacional que posteriormente se adoptan por el Estado mexicano y, de ahí, se implementan en la administración pública, misma de la que forman parte las universidades públicas.

En el plano federal, tales políticas con perspectiva de género datan de 1995 y llevaron a la creación del Instituto Nacional de la Mujer, de la Fiscalía Especializada para los Delitos contra las Mujeres y Trata de Personas, así como a la Ley General de Acceso a las Mujeres 
a una Vida Libre de Violencia (Zamudio-Sánchez, Andrade-Barrera, Arana-Ovalle, \& Alvaro-Segura, 2017). En México, los institutos y la ley en cuestión trazaron el itinerario de las políticas de equidad de género hasta la segunda década del siglo XXI. Este movimiento de cascada va en sentido contrario al trayecto seguido por la esfera internacional, donde los cambios vinieron desde los miembros de la sociedad, con movimientos desde la base (Barreto Ávila \& Flores Garrido, 2016).

Si bien los cambios normativos son pasos hacia la equidad, son insuficientes si no van acompañados por procesos más profundos que cuestionen el orden imperante y que identifiquen las condiciones reproductoras de las relaciones asimétricas de dominación propias de un sistema patriarcal (Varela Guinot, 2020). El aporte de Varela Guinot concuerda con el nivel epistemológico planteado por Palomar Verea. Esta investigadora señala la toma de decisiones respecto a las desigualdades entre géneros y en las estructuras jerarquías inequitativas como elemento que debe acompañar al diseño de políticas. La ausencia de un cambio profundo ha favorecido a una estructura social que se basa en la dominación masculina, en el uso de la violencia como principal potestad de quien está por encima de la otra persona (Varela Guinot, 2020).

Si la perspectiva feminista ha cuestionado la ética en el campo de la vida cotidiana, en una IES cabe cuestionarse sobre las prácticas institucionales que naturalizan el pensamiento binario y la implícita diferenciación de géneros. Este aspecto se pone de relieve en concursos de belleza, la presencia de edecanes mujeres en eventos académicos o el acoso sexual, por citar algunos ejemplos (Palomar Verea, 2005). Asimismo, hay una propensión a diluir la violencia con expresiones como: "así ha sido siempre" o, incluso, "es natural". Dichas locuciones normalizan y definen hábitos de comportamiento; abonan en una dinámica de minimización del problema con acuerdos y complicidades entre víctimas y victimarios que operan en la conservación de la hegemonía masculina (Lópes-Molina \& Vázquez-Guerrero, 2018).

Lo anterior está acompañado por la representación de las universidades como islas separadas, ajenas a los vicios del mundo exterior (Mingo \& Moreno, 2015). Esa imagen de los espacios universitarios lleva a diferentes tipos de prácticas para silenciar a las víctimas, entre los que sobresalen el hecho de tratar los casos como si fueran aislados y no como un problema estructural, las dificultades para conocer el impacto de los programas y las políticas que se encargan de atender la violencia en contra de las mujeres y la opacidad con que las instituciones manejan los diferentes tipos de violencia. El problema persiste bajo todo lo que conlleva esta percepción. El velo de la ignorancia evita fisuras en la forma idílica de entender la institución (Mingo \& Moreno, 2015; Varela Guinot, 2020).

Los factores enumerados forman parte de lo que Araceli Mingo y Hortensia Moreno denominan "ignorancia intencionada", es decir, un proceso sistemático de auto IQUAL. REVISTA DE GÉNERO E IGUALDAD, 2022, 5, 51-78 ISSN. 2603-851X DOI. http://dx.doi.org/10.6018/iqual.483711 
decepción: aquellos que ocupan un emplazamiento privilegiado abrazan intencionadamente la ignorancia, ignoran al opresor y el rol que juega la institución en las diferentes explotaciones (2015). Este hecho contribuye al silenciamiento de las mujeres víctimas del que habla Varela Guinot: al culparlas por factores relacionados con las formas de vestir, de hablar, de caminar, de bailar o de beber; al juzgarlas por las personas con quienes entablan relaciones de amistad o afectivas, tiene lugar una naturalización paulatina de los discursos que las representan como objetos en beneficio de la satisfacción de un supuesto impulso sexual incontrolable por parte de los hombres. Esto contribuye a la trivialización de la disconformidad que produce la violencia e incrementa los obstáculos y los costos que implican las quejas y las denuncias (Mingo \& Moreno, 2015).

Bajo este contexto surgen movilizaciones como La Red. Barreto Ávila y Flores Galindo (2016) señalan que se trata de una agrupación principalmente de estudiantes a la que se agregaron académicas y trabajadoras. El grupo se creó con la finalidad de exigir justicia en delitos de género ante autoridades universitarias, de seguridad pública y judicial. El motivo principal es que las denuncias de acoso y hostigamiento sexual se intensificaron en las IES en la última década (Mingo \& Moreno, 2015 y Varela Guinot, 2020). En ese mismo sentido, entre 2004 y 2018 se recopilaron 171 denuncias de acoso sexual en IES, principalmente en medios de comunicación. Sólo 7 se habían presentado antes de 2012, mientras que 109 correspondían a los dos últimos años (Varela Guinot, 2020). Esto permite afirmar que las víctimas se atreven a denunciar más frecuentemente y lo hacen con mayor confianza gracias a la existencia de círculos de protección institucionales, como comisiones de género, pero también de la sociedad civil y grupos feministas (Mingo \& Moreno, 2015; Barreto Ávila y Flores Galindo 2016; Varela Guinot, 2020).

En 2014 se realizó una encuesta sobre hostigamiento sexual sufrido por alumnos de la Universidad Nacional Autónoma de México (UNAM). En la mayoría de los casos los agresores eran hombres; la mayoría de las víctimas no hicieron nada al respecto, sólo un pequeño porcentaje de las víctimas denunciaron ante autoridades institucionales (Mingo \& Moreno, 2015). Tres casos paradigmáticos tuvieron lugar en la misma UNAM: hubo manifestaciones en público como forma de ejercer presión y que el rector reaccionara contra victimarios de delitos de género (Barreto Ávila \& Flores Garrido, 2016). Tales acciones, en la cuales participó activamente La Red, tuvieron resonancia dentro y fuera de la universidad (Mingo \& Moreno, 2015; Barreto Ávila \& Flores Garrido, 2016). En el exterior, acudieron ante la CNDH a poner una queja por violencia sexual y consiguieron que el organismo emitiera una recomendación a la universidad (Mingo \& Moreno, 2015). Varios miembros del consejo la calificaron como infundada, engañosa y que pretendía desacreditar tanto al organismo como a las autoridades del mismo, lo que puso al 
descubierto vacíos institucionales para atender casos de denuncia por violencia de género (Mingo \& Moreno, 2015; Barreto Ávila \& Flores Garrido, 2016).

En una universidad especializada en ciencias agropecuarias se realizó un estudio que mostró que la violencia de género es estructural, ya que las estudiantes manifestaron la existencia evidente de delitos dentro de la institución que no son sancionados: las instancias disciplinarias no aplican la normatividad, lo anterior debido, de acuerdo a los testimonios de las personas participantes, a la existencia de abuso de poder, corrupción e impunidad entre las mismas autoridades que son mayoritariamente hombres (ZamudioSánchez, Andrade-Barrera, Arana-Ovalle, \& Alvaro-Segura, 2017). El principal delito mencionado fue el acoso sexual, el cual es padecido por las mujeres de manera constante por parte de los hombres. Esto genera un problema de impunidad que permea a la sociedad a causa de que, al no ejercerse apropiadamente la justicia, las personas se desalientan para defender sus derechos (Ibídem).

Por otra parte, lo ocurrido en la UNAM puso de manifiesto que la erradicación de la violencia de género en universidades sólo se puede lograr mediante la institucionalización de la perspectiva de género (Barreto Ávila \& Flores Garrido, 2016). De acuerdo con las estudiantes víctimas, el apoyo y la solidaridad recibida principalmente por parte de la comunidad feminista universitaria, fueron fundamentales para transformar el duelo y la culpa en la indignación y el enojo necesarios para sacar a la luz sus casos en una carta que circuló entre la comunidad (Mingo \& Moreno, 2015). De igual manera, ha quedado al descubierto que en la universidad no han existido ejercicios que partan de metodologías participativas para formular políticas con perspectiva de género (Mingo \& Moreno, 2015).

Un artículo de Cortazar Rodríguez sobre hostigamiento y acoso sexual padecido por estudiantes se centra en la Universidad de Guadalajara. Las víctimas expresaron que para levantar denuncias les solicitan pruebas difíciles de conseguir, los casos se dan en el ámbito privado, de manera verbal y no hay testigos; además, ante la falta de protocolos y vacíos legislativos, las autoridades universitarias no saben cómo proceder. Ese mismo estudio ofrece como ejemplo el caso de un alumno acosador denunciado. Lo canalizaron a consulta psicológica, fue diagnosticado con un trastorno y las autoridades universitarias no pudieron evitar su incidencia en futuras conductas de acoso y hostigamiento. Por lo que el procedimiento, cuando hay denuncia, se percibe como desigual entre las partes: se les cita a ambas a un careo, hecho que propicia la re-victimización y la culpa, la imputación a la víctima de haber incitado al hostigamiento. Esta forma de proceder contribuye a que los casos no se denuncien (Cortazar Rodríguez, 2020).

Son pocas las universidades que han diseñado algún protocolo o mecanismo de acción para enfrentar el problema de violencia de género al interior de las instituciones (Mingo \& Moreno, 2015; Barreto Ávila \& Flores Garrido, 2016; Briseño Maas \& Bernabé IQUAL. REVISTA DE GÉNERO E IGUALDAD, 2022, 5, 51-78 ISSN. 2603-851X DOI. http://dx.doi.org/10.6018/iqual.483711 
Morales, 2019; Varela Guinot, 2020). La implementación de protocolos de atención a la violencia de género pudiera ser la respuesta a los reclamos que señalan la falta de capacidad por parte de las autoridades para dar respuesta oportuna y eficiente a los actos de violencia de género dentro de las IES (Briseño Maas \& Bernabé Morales, 2019). Pero es relevante tener en consideración que los protocolos de atención a víctimas requieren de cursos de sensibilización y capacitación para toda la comunidad universitaria, así como de información sobre los derechos y deberes de cada miembro (Cortazar Rodríguez, 2019). Esto debido a que el alumnado no sabe ante quien acudir para presentar la denuncia, la ruta a seguir ni de sus derechos (Cortazar Rodríguez, 2019).

Debido a lo anterior es que, desde la academia, particularmente la feminista, se señala la necesidad de institucionalizar la igualdad de género como un marco de sentido que oriente las interacciones sociales (Barreto Ávila \& Flores Garrido, 2016). Con los estudios analizados en estas líneas se evidencia la vigorización de la intolerancia hacia el sexismo y la violencia de género; por lo que se puede hablar, de acuerdo con Moreno y Mingo (2020), de un proceso de alteración profunda de la cultura machista dominante observado en: conductas que en el siglo XX eran consideradas corrientes y aceptables, ahora son insostenibles; así como la señalización del escandaloso desequilibrio de poder, la inequidad y la vileza presente en las conductas y disposiciones de un número significativo de varones para con las mujeres principalmente.

En México el contar con protocolos de atención para casos de violencia de género escasea. Según Briseño Maas \& Bernabé Morales (2019), eran 10 IES, 8 públicas y 2 privadas. Para finales de 2018 eran sólo 15 universidades mexicanas las que contaban con protocolos de actuación para casos de hostigamiento y acoso sexual (Cortazar Rodríguez, 2019). Una segunda evidencia más en profundidad sobre las políticas para atender estos problemas es el trabajo de Varela Guinot (2020) que analizó tres aspectos:

- El primero, si se han incluido medidas específicas para atender los casos.

- Segundo, quiénes son las autoridades encargadas de atender los casos.

- Tercero, cómo de accesibles es la información sobre los instrumentos existentes.

Cuyos resultados permitieron clasificar a las instituciones en tres tipos:

- Protocolo, instituciones que cuentan con un instrumento, ámbitos de aplicación, alcance, instancias responsables y ruta crítica para seguir la denuncia ( $28 \%$ de las instituciones de su muestra).

- Protocolo Parcial, instituciones con instrumento, pero que tienen alguna deficiencia como no atender a toda la comunidad universitaria, no se pudo 
acceder al documento, se limita a dar indicaciones generales o cuando no abarca a toda la institución (23\%).

- Sin Protocolo, las que carecen de instrumento para atender casos de violencia de género (49\%).

En los casos de existir protocolos la creación de estos se ha enfrentado a procesos sumamente complicados, como la escasa atención por parte de los tomadores de decisiones (Briseño Maas \& Bernabé Morales, 2019). En cuanto a las instituciones con protocolos que se analizaron las principales carencias fueron: la falta de restricciones y sanciones para infractores no graves, o que la investigación pueda revertirse en caso de que se presuma que la denunciante se condujo con dolo por falsedad o pruebas falsas (Ibídem).

En ese mismo sentido, la investigación de Varela Guinot (2020) tomó como variable el quién hace la investigación y quien emite la resolución, las consideró importante porque reflejan el modo en que la institución percibe la problemática de la violencia de género y hasta qué punto se entiende que debe haber instancias especiales que conozcan de la materia para atender los casos; también porque en la medida con que se cuente con personas capacitadas y no involucradas directamente con los casos denunciados, los riesgos de la discrecionalidad disminuyen notablemente.

Por lo tanto, concluye Varela Guinot (2020) que el deber respecto a la atención de estas problemáticas por parte de las IES es garantizar espacios de deliberación sensibles a la problemática, con capacidad de actuar libremente y autónomamente; así como la solución contra la violencia de género desde esta perspectiva estaría en propiciar formas de relación que no estén basadas en la dominación e imposición. Por lo que, para finalizar el estudio de Varela Guinot, las instituciones más que definir mecanismos para hacer frente a estos casos deben encarar las condiciones estructurales que las propician.

Los antecedentes para México aquí expuestos muestran que principalmente el problema se ha atacado de abajo hacia arriba, es decir, por medio de los reclamos organizados de la población afectada. Debido a la mayor denuncia pública de casos de hostigamiento sexual, las investigadoras Mingo y Moreno (2020) expresan un cuestionamiento respecto a si se han incrementado los comportamientos hostiles de los hombres hacia las mujeres o si se ha acentuado la sensibilidad de las mujeres hacia las agresiones de los hombres.

Es así que la exigencia hacia el nivel institucional va en el sentido de la erradicación del sexismo. Este último, entendido por Mingo y Moreno (2020) como expresiones variadas de violencia que están en la raíz de las relaciones jerárquicas instituidas entre los sexos y

IQUAL. REVISTA DE GÉNERO E IGUALDAD, 2022, 5, 51-78

ISSN. 2603-851X

DOI. http://dx.doi.org/10.6018/iqual.483711 
dispositivos de poder con que se pretende mantener el orden de género en las interacciones cotidianas.

\section{Marco analítico}

Los entes sociales, como lo son las universidades, contienen acciones sociales que vistas desde la perspectiva de Weber son conductas socialmente orientadas de un número determinado de individuos (2004). Estos individuos tienen roles distintos dentro de la organización, están los gobernantes, los funcionarios administrativos, los académicos y los estudiantes. Estas personas conforman un entramado social jerárquico con relaciones de poder. Una problemática en tales redes es el hecho que son personas con identidades de género, además de la posición jerárquica que ocupan.

Las acciones sociales se manifiestan como lucha social cuando se busca imponer una voluntad contra la resistencia de otros (Weber, 2004). Es así que esas relaciones devenidas de la lucha buscan un cambio pacífico o violento y, por lo tanto, son relaciones solidarias. De acuerdo con el mismo Weber, son acciones comunitarias relativas. Esto deja como evidencia la existencia de pugnas constantes entre quienes integran a las organizaciones. Luchas y resistencias de poder y dominación.

La dominación se legitima cuando se pacta o cuando se otorga por una autoridad considerada como legítima (Weber, 2004). Es así como se tiene un orden social que se hará respetar coactivamente por "cuadros de individuos instituidos con la obligación de observar dicho orden" (Ibídem, p. 27). Por lo cual es un orden de derecho.

El problema con respecto al orden de las relaciones sociales es que hay discursos de poder que se materializan en el cuerpo. En éste, de acuerdo con Butler (2002), el cuerpo domina al espíritu, lo material impone cuerpo sobre alma; es así que se ha materializado el cuerpo femenino como lo excluido, la otredad al masculino, excluido de la economía y privado de esencia.

Estos discursos se realizan, en términos de Butler (2002), en representaciones teatrales de la cotidianeidad que son transferibles y plásticos. Por lo que se construyen desde sus concepciones, se producen y reproducen en la vida cotidiana, por lo tanto, no son estáticos sino performativos. Hay una relación entre el cuerpo y su significado. De acuerdo al mismo texto de Butler, el cuerpo no es pasivo es una demanda de acción, la continuidad de la psique.

Esta materia es una instrumentalización y un despliegue de una serie de relaciones lingüísticas más amplias. El cuerpo es discurso, afirma Butler, su lenguaje surge de la materialidad de la vida corporal, como la reiteración y la extensión de las relaciones, el 
lenguaje es un acto de desplazamiento y condensación. Lo que constituye a la materialidad del discurso es su iteración.

Pero las identificaciones nunca se hacen o se alcanzan definitivamente, se les negocia insistentemente (Butler, 2002). Incluso se convierten en luchas sociales, en las que el rechazo a la ley, cuando el sector de la sociedad afectado acrecienta su poder, debido a que la capacidad de acción está implicada en relaciones de poder, obliga a su rearticulación y pone en tela de juicio su fuerza unilateral (Ibídem).

Es especialmente relevante distinguir la política de lo político. La política atañe a un sistema de gobierno, partidos políticos y la política cotidiana, mientras lo político es dimensión instituyente y fundacional de la sociedad. También se presenta lo segundo como desfundacional cuando se dan crisis democráticas que tienen que ver con "la disolución de razones finales exclusivas e inamovibles como el Estado, el mercado, la clase, pero también la libertad o el género" (Wolfesberger, 2019, p. 46).

Una crítica que expresa Wolfesberger (2019) al individualismo como se ha presentado en la filosofía occidental es la ausencia del ser humano en su pluralidad, es decir la acción política conjunta de varias personas. La cual tiene su validez en la lucha social que es colectiva y de grupos que se presentan en condiciones de desigualdad y discriminación, el texto de Wolfesberger está situado en las comunidades indígenas o de pueblos originarios mexicanos, pero la crítica también se aplica como él mismo lo indica por razones de género a los movimientos feministas. Por lo tanto, una democracia que tome en cuenta a la comunalidad orienta la solución hacia la inclusión de diferentes puntos de vista (Wolfesberger, 2019).

\section{Metodología}

El objetivo de este trabajo es analizar las medidas contra la violencia de género en Instituciones de Educación Superior. Para lograrlo se tomó como fuente de información la que ofrece el Observatorio Nacional para la Igualdad de Género en las Instituciones de Educación Superior (ONIGIES). A partir de esa información se buscó responder a la siguiente pregunta: ¿Cuál es la reacción que las IES han tenido ante la necesidad de atender casos de violencia de género dentro de sus instituciones?

ONIGIES es un proyecto colectivo en el que participan organismos públicos de derechos humanos y de derechos de las mujeres, así como centros de las IES dedicados a temas de género. Tiene la intensión explícita de crear espacios académicos libres de desigualdad por razones de género (ONIGIES, 2020). Para lograr tal fin crearon un índice

IQUAL. REVISTA DE GÉNERO E IGUALDAD, 2022, 5, 51-78

ISSN. 2603-851X

DOI. http://dx.doi.org/10.6018/iqual.483711 
de igualdad de género en las IES para medir el avance de esas instituciones a partir de 2017, año del cual se tenía disponible información.

El citado índice comprende ocho ejes temáticos que son:

1. Legislación con perspectiva de género, comprende a la normatividad institucional, órganos de igualdad de género, planes de igualdad de género y los recursos financieros para el impulso de la igualdad de género.

2. Corresponsabilidad familiar, comprendida en programas.

3. Estadísticas y diagnóstico con perspectiva de género que son: bases de datos e informes con perspectiva de género, así como encuestas y diagnósticos con perspectiva de género.

4. Lenguaje incluyente y no sexista.

5. Sensibilización en género que abarca educación en igualdad de género y la sensibilización en género.

6. Investigación y estudios de género comprende, cursos curriculares en estudios de género e investigación con perspectiva de género.

7. Violencia de género que es: atención de casos de violencia de género y prevención a la violencia de género.

8. Igualdad de oportunidades observada en la distribución por sexo en la población estudiantil, personal académico y administrativo, así como en las autoridades.

Con la información que se recaba en cada eje se realiza una calificación que consiste en: dos puntos para valorar la institucionalización de políticas para estos fines y tres puntos para la transversalización de esas mismas políticas en las entidades académicas y administrativas de las IES (ONIGIES, 2020).

La información se sistematizó para su análisis en dos grupos de categorías. La primera, institucionalización que comprende las acciones específicas en cada IES por eje. La segunda, transversalización que comprende la cantidad de entidades académicas y administrativas que llevan a cabo dichas acciones. También se sistematizó información demográfica de las IES en su matrícula de licenciatura, personal académico y administrativo, así como en su máxima autoridad colegiada.

Se seleccionaron las siete IES con mayor matrícula de licenciatura, debido a que la matrícula de nivel superior se ubica principalmente ahí, que proporcionaran información al Observatorio Nacional para la Igualdad de Género en las Instituciones de Educación Superior (ONIGIES), así como se tomó al año de referencia 2107 que es el año más 
reciente con información de ONIGIES. Para los datos sobre matrícula se recurrió a los anuarios estadísticos de la ANUIES. Posteriormente se pasó a una comparación demográfica de las instituciones, por composición de acuerdo a la variable sexo en los cuerpos colegiados máximos, personal académico, administrativo y población estudiantil en licenciatura. Por último, se describió cada IE en las categorías tomadas por ONIGIES para calificar la institucionalización de procesos con perspectiva de género.

Lo anterior tiene la intención de ofrecer un panorama desde las instituciones más grandes en México, federales y estatales, sobre los siete ejes temáticos para la igualdad de género. También en el ejercicio se compara a estas instituciones en los programas y políticas emprendidos para dichos aspectos básicos para una vida institucional aceptable. De las diez universidades con más matrícula, siete participan en ONIGIES.

\section{Resultados}

\subsection{Descripción demográfica de la IES}

Las siete universidades con más matrícula en licenciatura, de acuerdo a los anuarios estadísticos de ANUIES son dos Federales: UNAM e IPN (Instituto Politécnico Nacional); así como cinco estatales: Universidad de Guadalajara (UDG), Universidad Autónoma de Sinaloa (UAS), Benemérita Universidad Autónoma de Puebla (BUAP), Universidad Veracruzana (UV) y Universidad Autónoma del Estado de México (UAEM). Con el siguiente orden de mayor a menor:

Tabla 1

Matrícula por sexo

\begin{tabular}{|c|c|c|c|}
\hline IE & Hombres & Mujeres & Total \\
\hline UNAM & 63,254 & 70,448 & 133,702 \\
\hline UDG & 54,749 & 60,975 & 115,724 \\
\hline IPN & 60,911 & 39,479 & 100,390 \\
\hline UAS & 36,716 & 42,371 & 79,087 \\
\hline BUAP & 34,866 & 40,188 & 75,054 \\
\hline UV & 28,599 & 31,982 & 60,581 \\
\hline UAEM & 25,269 & 32,887 & 58,156 \\
\hline
\end{tabular}

Fuente: Anuario estadístico ANUIES 2017. 
Por lo que respecta a la distribución por sexo, en la mayoría de las instituciones, a excepción del IPN, la matrícula estaba cercana a la paridad, ligeramente eran mayoría las mujeres (ver Gráfica 1). 


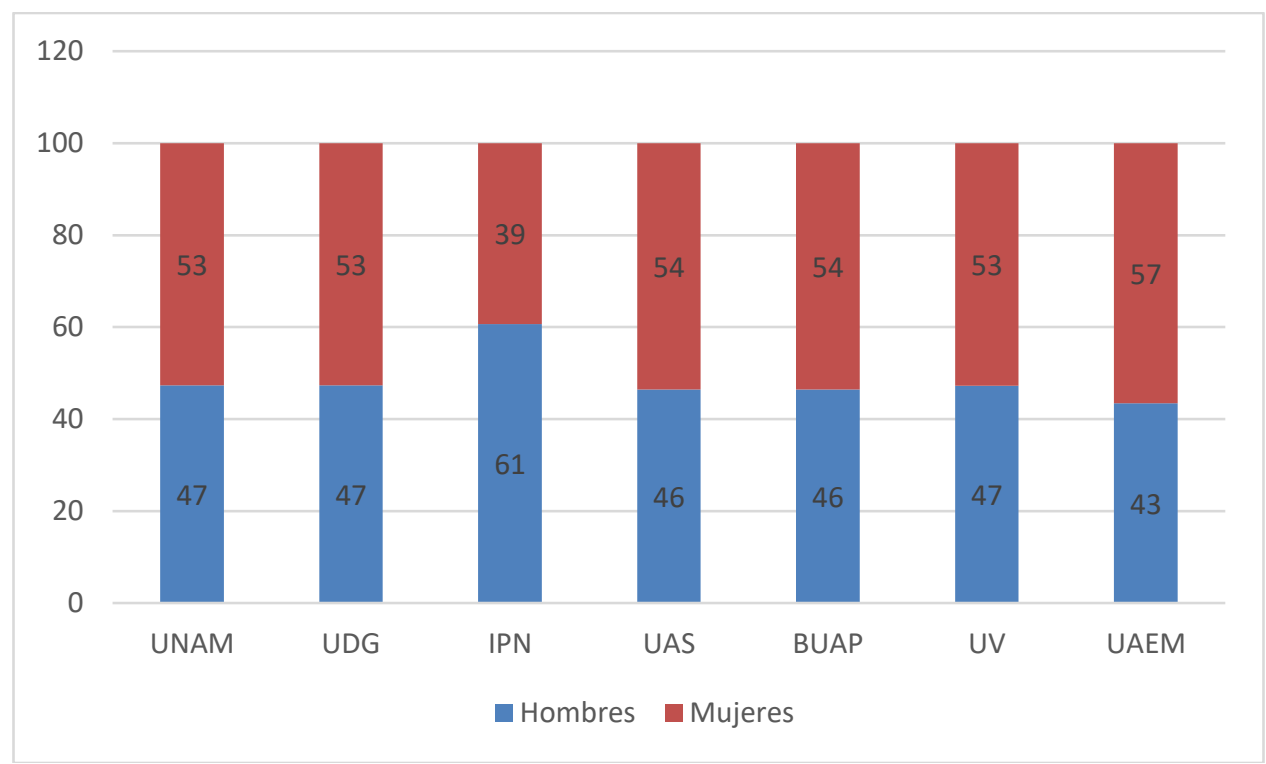

Figura 1. Distribución de la matrícula por sexo.

Fuente: Anuario estadístico ANUIES 2017.

Respecto a autoridades, en el máximo cuerpo colegiado, la proporción por sexo muestra que en UDG e IPN la mayoría de miembros de la máxima autoridad son hombres, en la UNAM y la UV la mayoría de hombres disminuye, en la BUAP y UAEM la proporción está equilibrada y la UAS es la única universidad en las mujeres superan el 50\% (ver Gráfica 2).

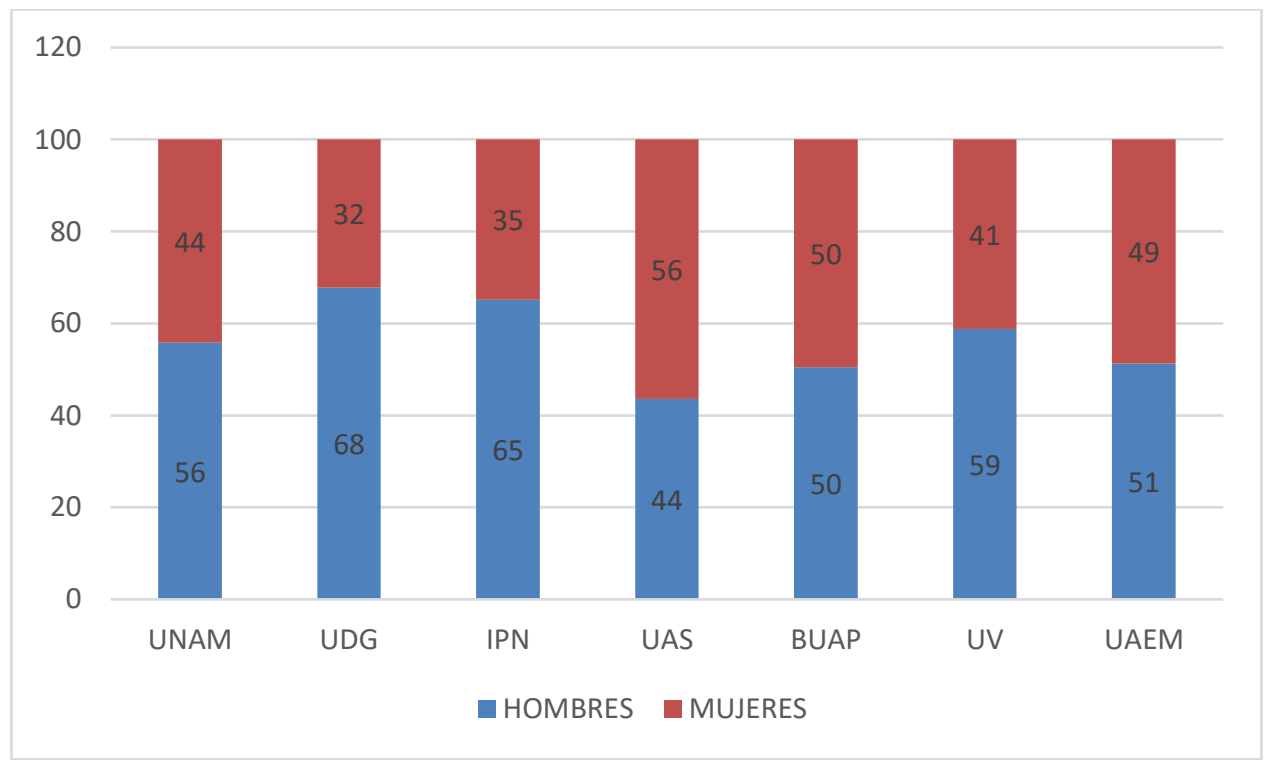

Gráfica 2. Proporción hombres/mujeres máximo cuerpo colegiado.

Fuente: ONIGIES 2017.

En cuanto a la proporción de académicos de tiempo completo, en todas las siete instituciones estudiadas las mujeres se encontraban por debajo del 50\%. En la UNAM y la

IQUAL. REVISTA DE GÉNERO E IGUALDAD, 2022, 5, 51-78

ISSN. 2603-851X

DOI. http://dx.doi.org/10.6018/iqual.483711 
BUAP la proporción era del 45\%. Mientras que en el IPN y la UAS no llegaban al 40\%. En las otras tres UDG, UAEM y UV representaban el 42\% (ver Gráfica 3).

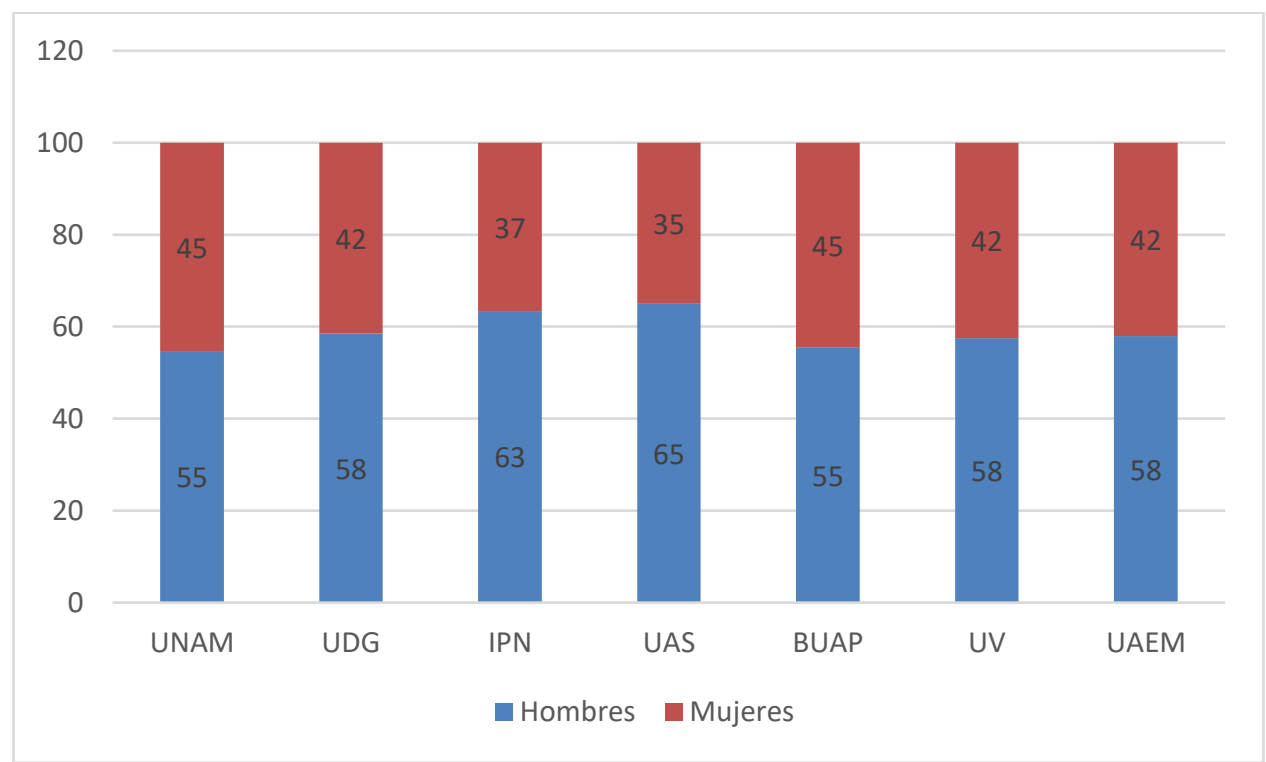

Figura 3. Proporción de académicos de tiempo completo por sexo.

Fuente: ONIGIES 2017.

En cuanto al personal administrativo de base en todas las instituciones la distribución por sexo está equilibrada, únicamente en la UV la mayoría del personal son mujeres (ver Gráfica 4).

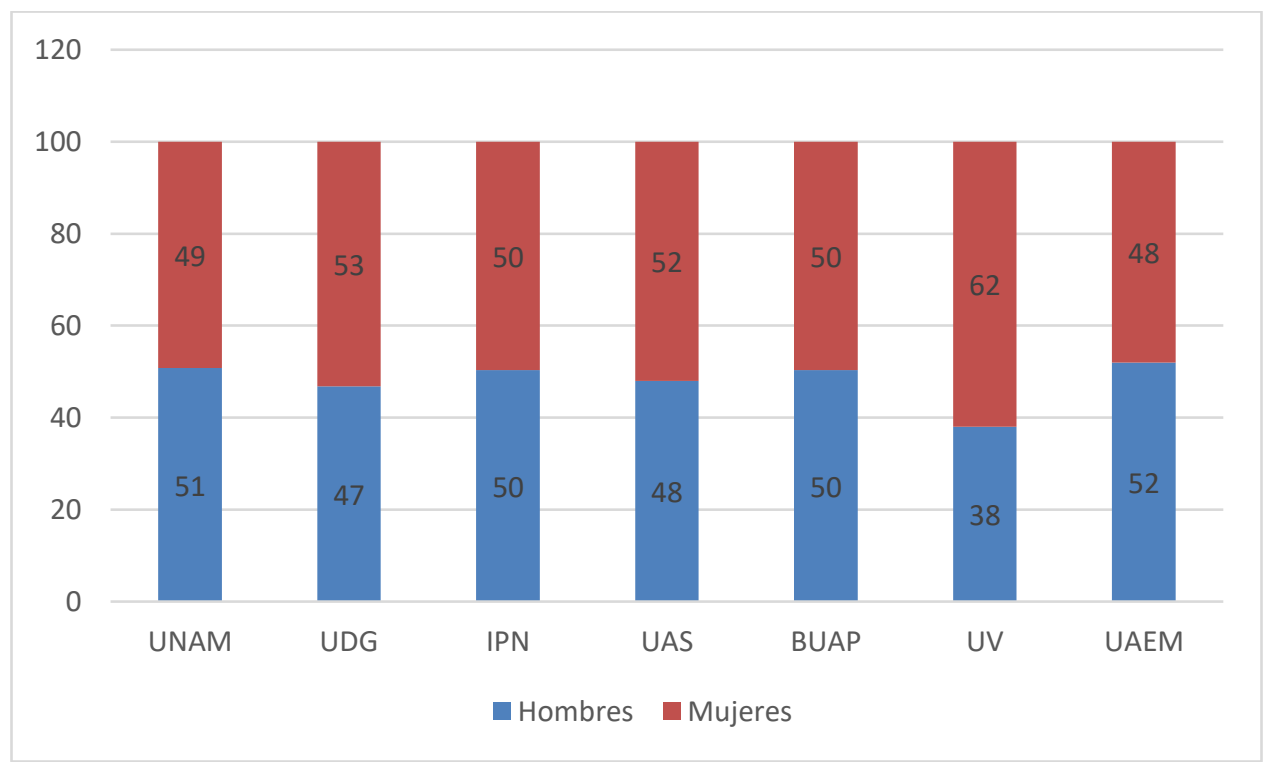

Figura 4. Proporción del personal administrativo por sexo.

Fuente: ONIGIES 2017. 


\subsection{Análisis ejes igualdad de género}

\subsubsection{Institucionalización}

El índice de igualdad de género de ONIGIES tiene un puntaje máximo de 5, para 2017 el índice de las IES en México era de 1.5, como se observó en su página de Internet. En este apartado se hará un análisis en cada uno de los siete ejes en las universidades objeto de este estudio.

La UNAM tiene un puntaje de ONIGIES de 2.4 en general. A nivel de institucionalización de cada uno de los ejes el estado que guarda por acciones es el siguiente:

- Primero, Estadísticas y Diagnósticos con Enfoque de Género, cuenta con un anuario estadístico oficial (o documento afín) con información desagregada por sexo sobre toda su estructura.

- Segundo, Investigaciones y Estudios de Género, tiene un área o línea de conocimiento en estudios de género que es el centro que participa en el observatorio, así como una instancia con recursos y personal propios para la investigación académica con perspectiva de género.

- Tercero, Legislación con Perspectiva de Género, la institución reconoce explícitamente el principio de igualdad de género en su máximo documento normativo, cuenta con un documento de normas oficiales para la igualdad de género, tiene un órgano que emite periódicamente medidas y recomendaciones de igualdad de género vinculantes para toda la institución y tuvo en su presupuesto anual recursos específicos etiquetados para la igualdad de género.

- Cuarto, Lenguaje Incluyente y no Sexista, redacta en femenino en todos los títulos profesionales otorgados a mujeres, así como un documento oficial para un uso incluyente y no sexista del lenguaje en sus comunicaciones institucionales.

- Quinto, No Violencia, tiene un instrumento para Proceder frente a casos de violencia de género y un programa oficial de actividades para la prevención de la violencia de género.

- Sexto, Sensibilización en Género, tuvo un programa permanente de cursos y/o talleres extracurriculares sobre género para toda su comunidad y una campaña para promover la igualdad de género en toda su comunidad.

La segunda universidad en tamaño de matrícula, UDG, tiene un puntaje en el índice de o.9 en términos de institucionalización los indicadores que cumple son los siguientes:

IQUAL. REVISTA DE GÉNERO E IGUALDAD, 2022, 5, 51-78

ISSN. 2603-851X

DOI. http://dx.doi.org/10.6018/iqual.483711 
- Primero, Estadísticas y Diagnósticos con Enfoque de Género, cuenta con un anuario estadístico oficial (o documento afín) con información desagregada por sexo sobre toda su estructura.

- Segundo, Investigación y Estudios de Género, tiene un área o línea de conocimiento en estudios de género, así como una instancia con recursos y personal propios para la investigación académica con perspectiva de género.

- En los demás ejes no cuenta con ningún indicador.

La siguiente institución es el IPN con un puntaje en el índice de 1.3 y cumple con los siguientes indicadores por eje:

- Primero, Corresponsabilidad Familiar, si incluye dentro de su política estrategias para promover la corresponsabilidad.

- Segundo, Estadísticas y Diagnósticos con Enfoque de Género, cuenta con un anuario estadístico oficial (o documento afín) con información desagregada por sexo sobre toda su estructura.

- Tercero, Investigación y Estudios de Género, tiene un área o línea de conocimiento en estudios de género, así como una instancia con recursos y personal propios para la investigación académica con perspectiva de género.

- Cuarto, Legislación con Perspectiva de Género, tiene un órgano que emite periódicamente medidas y recomendaciones de igualdad de género vinculantes para toda la institución, acciones a favor de la igualdad de género en el programa de trabajo de la máxima autoridad de la institución y tuvo en su presupuesto anual recursos específicos etiquetados para la igualdad de género.

- Quinto, Lenguaje Incluyente y no Sexista, promocionaron el uso de un lenguaje incluyente en toda su comunidad y redacta en femenino en todos los títulos profesionales otorgados a mujeres.

- Sexto, Sensibilización en Género, tuvo un programa permanente de cursos y/o talleres extracurriculares sobre género para toda su comunidad

La cuarta IES es la UAS que tiene un puntaje en el índice de 2.4 , en cuanto a los siete ejes su estado es el siguiente:

- Primero, Estadísticas y Diagnósticos con Enfoque de Género, cuenta con un anuario estadístico oficial (o documento afín) con información desagregada por sexo sobre toda su estructura. 
- Segundo, Investigaciones y Estudios de Género, tiene un área o línea de conocimiento en estudios de género que es el centro que participa en el observatorio, así como una instancia con recursos y personal propios para la investigación académica con perspectiva de género.

- Tercero, Legislación con Perspectiva de Género, la institución reconoce explícitamente el principio de igualdad de género en su máximo documento normativo, cuenta con un documento de normas oficiales para la igualdad de género, tiene un órgano que emita periódicamente medidas y recomendaciones de igualdad de género vinculantes para toda la institución, tuvo en su presupuesto anual recursos específicos etiquetados para la igualdad de género, así como una instancia administrativa con recursos y personal propios para implementar estrategias generales para la igualdad de género en toda la institución.

- Cuarto, Lenguaje Incluyente y no Sexista, promocionaron el uso de un lenguaje incluyente en toda su comunidad y redacta en femenino en todos los títulos profesionales otorgados a mujeres.

- Quinto, No Violencia, tiene un instrumento para proceder frente a casos de violencia de género y un programa oficial de actividades para la prevención de la violencia de género.

- Sexto, Sensibilización en Género, tuvo un programa permanente de cursos y/o talleres extracurriculares sobre género para toda su comunidad y una campaña para promover la igualdad de género en toda su comunidad.

Por lo que respecta a la BUAP tiene un puntaje en el índice de 1.3 , en los siete ejes contaba con los siguientes indicadores:

- Primero, Estadísticas y Diagnósticos con Enfoque de Género, cuenta con un anuario estadístico oficial (o documento afín) con información desagregada por sexo sobre toda su estructura.

- Segundo, Investigaciones y Estudios de Género, una instancia con recursos y personal propios para la investigación académica con perspectiva de género.

- Tercero, Legislación con Perspectiva de Género, tiene un órgano que emite periódicamente medidas y recomendaciones de igualdad de género vinculantes para toda la institución, una instancia administrativa con recursos y personal propios para implementar estrategias generales para la igualdad de género en toda la institución y acciones a favor de la igualdad de género en el programa de trabajo de la máxima autoridad de la institución.

IQUAL. REVISTA DE GÉNERO E IGUALDAD, 2022, 5, 51-78

ISSN. 2603-851X

DOI. http://dx.doi.org/10.6018/iqual.483711 
- Cuarto, Lenguaje Incluyente y no Sexista, promocionaron el uso de un lenguaje incluyente en toda su comunidad y redacta en femenino en todos los títulos profesionales otorgados a mujeres.

- Quinto, Sensibilización en Género, tuvo un programa permanente de cursos y/o talleres extracurriculares sobre género para toda su comunidad y una campaña para promover la igualdad de género en toda su comunidad.

La penúltima IES dentro de este análisis es la UV que cuenta con un puntaje de 2.3 en el índice, contaron con los siguientes indicadores en los ejes de ONIGIES:

- Primero, Estadísticas y Diagnósticos con Enfoque de Género, cuenta con un anuario estadístico oficial (o documento afín) con información desagregada por sexo sobre toda su estructura.

- Segundo, Investigaciones y Estudios de Género, tiene un área o línea de conocimiento en estudios de género, así como una instancia con recursos y personal propios para la investigación académica con perspectiva de género.

- Tercero, Legislación con Perspectiva de Género, la institución reconoce explícitamente el principio de igualdad de género en su máximo documento normativo, cuenta con un documento de normas oficiales para la igualdad de género, tiene un órgano que emite periódicamente medidas y recomendaciones de igualdad de género vinculantes para toda la institución, instancia administrativa con recursos y personal propios para implementar estrategias generales para la igualdad de género en toda la institución, acciones a favor de la igualdad de género en el programa de trabajo de la máxima autoridad de la institución y tuvo en su presupuesto anual recursos específicos etiquetados para la igualdad de género.

- Cuarto, Lenguaje Incluyente y no Sexista, promocionaron el uso de un lenguaje incluyente en toda su comunidad y redacta en femenino en todos los títulos profesionales otorgados a mujeres.

- Quinto, No Violencia, tiene un instrumento para proceder frente a casos de violencia de género y un programa oficial de actividades para la prevención de la violencia de género.

- Sexto, Sensibilización en Género, tuvo un programa permanente de cursos y/o talleres extracurriculares sobre género para toda su comunidad y una campaña para promover la igualdad de género en toda su comunidad.

Por último, la UAEM tuvo un puntaje de 1.2 en el índice, así como contaba con los siguientes indicadores:

IQUAL. REVISTA DE GÉNERO E IGUALDAD, 2022, 5, 51-78 ISSN. 2603-851X DOI. http://dx.doi.org/10.6018/iqual.483711 
- Primero, Estadísticas y Diagnósticos con Enfoque de Género, cuenta con un anuario estadístico oficial (o documento afín) con información desagregada por sexo sobre toda su estructura.

- Segundo, Investigaciones y Estudios de Género, tiene un área o línea de conocimiento en estudios de género, así como una instancia con recursos y personal propios para la investigación académica con perspectiva de género.

- Tercero, Legislación con Perspectiva de Género, contaba con una instancia administrativa con recursos y personal propios para implementar estrategias generales para la Igualdad de Género en toda la Institución, así como acciones a favor de la igualdad de género en el programa de trabajo de la máxima autoridad de la institución.

- Cuarto, Lenguaje Incluyente y no Sexista, promocionaron el uso de un lenguaje incluyente en toda su comunidad.

- Quinto, Sensibilización en Género, tuvo una campaña para promover la igualdad de género en toda su comunidad.

\subsubsection{Transversalización}

Las IES en esta dimensión de análisis aportaron información sobre la cantidad de entidades académicas y administrativas que realizaron 10 acciones dentro de los siete ejes. La UNAM tuvo un promedio total de 46.3 entidades. De las cuales, el de académicas fue 35 y administrativas 11.3. Las acciones se llevaron a cabo por eje de la siguiente manera: Corresponsabilidad Familiar, una en Estadísticas y Diagnósticos con Enfoque de Género, una en Investigaciones y Estudios de Género, tres en Legislación con Perspectiva de Género, dos en No Violencia y dos en Sensibilización en Género. De resaltar que la acción que más entidades, tanto administrativas como académicas, llevan a cabo es la de contar con mecanismos para presentar quejas por violencia de género (ver Tabla 2).

Tabla 2

Entidades de la UNAM que realizan acciones de igualdad de género

\begin{tabular}{|l|c|c|c|}
\hline \multicolumn{1}{|c|}{ ACCIONES } & ACADÉMICAS & ADMINISTRATIVAS & TOTAL \\
\hline $\begin{array}{l}\text { Realizaron acciones } \\
\text { explícitas para promover la } \\
\text { corresponsabilidad }\end{array}$ & 1 & 0 & 1 \\
\hline $\begin{array}{l}\text { Publicaron informes de } \\
\text { labores con datos } \\
\text { desagregados por sexo. }\end{array}$ & 42 & 0 & 42 \\
\hline Cuentan con grupos & 22 & 0 & 22 \\
\hline
\end{tabular}

IQUAL. REVISTA DE GÉNERO E IGUALDAD, 2022, 5, 51-78

ISSN. 2603-851X

DOI. http://dx.doi.org/10.6018/iqual.483711 


\begin{tabular}{|l|c|c|c|}
\hline $\begin{array}{l}\text { académicos con } \\
\text { perspectiva de género }\end{array}$ & 31 & 0 & 31 \\
\hline $\begin{array}{l}\text { Órganos internos para la } \\
\text { igualdad de género }\end{array}$ & 29 & 5 & 34 \\
\hline $\begin{array}{l}\text { Incluyen acciones a favor } \\
\text { de la igualdad de género en } \\
\text { los programas de trabajo } \\
\text { de sus titulares }\end{array}$ & 21 & 10 & 31 \\
\hline $\begin{array}{l}\text { Ejercieron recursos } \\
\text { económicos para la } \\
\text { igualdad de género }\end{array}$ & 103 & 61 & 164 \\
\hline $\begin{array}{l}\text { Cuentan con mecanismos } \\
\text { para la presentación de } \\
\text { quejas por violencia de } \\
\text { género }\end{array}$ & 26 & 7 & 33 \\
\hline $\begin{array}{l}\text { Realizaron actividades de } \\
\text { prevención de la violencia } \\
\text { de género }\end{array}$ & 26 & 82 & 34 \\
\hline $\begin{array}{l}\text { Realizaron cursos y/o } \\
\text { talleres con perspectiva de } \\
\text { género }\end{array}$ & & & \\
\hline $\begin{array}{l}\text { Realizaron actividades de } \\
\text { sensibilización para la } \\
\text { igualdad de género }\end{array}$ & 49 & & \\
\hline
\end{tabular}

Fuente: ONIGIES, 2017.

Con relación a la UDG, su promedio de entidades académicas y administrativas era de 7.16. Por tipo de entidad, el promedio de entidades académicas 7.16, mientras que el de administrativas era o. Por eje esa institución tenía dos acciones en el de Estadísticas y Diagnósticos con Enfoque de Género, uno en Legislación con Perspectiva de Género, uno en No violencia y dos en Sensibilización en género. El eje en el que más entidades cuentan con acciones el de Estadística y Diagnósticos con Enfoque de Género (ver tabla 3).

Tabla 3

Entidades de la UDG que realizan acciones de igualdad de género

\begin{tabular}{|l|c|c|c|}
\hline \multicolumn{1}{|c|}{ ACCIONES } & ACADÉMICAS & ADMINISTRATIVAS & TOTAL \\
\hline $\begin{array}{l}\text { Publicaron informes de } \\
\text { labores con datos } \\
\text { desagregados por sexo. }\end{array}$ & 12 & 0 & 12 \\
\hline Cuentan con & 16 & 0 & 16 \\
\hline
\end{tabular}

IQUAL. REVISTA DE GÉNERO E IGUALDAD, 2022, 5, 51-78 ISSN. 2603-851X DOI. http://dx.doi.org/10.6018/iqual.483711 


\begin{tabular}{|l|c|c|c|}
\hline $\begin{array}{l}\text { diagnósticos } \\
\text { cuantitativos de } \\
\text { igualdad de género }\end{array}$ & 1 & 0 & 1 \\
\hline $\begin{array}{l}\text { Órganos internos para la } \\
\text { igualdad de género }\end{array}$ & 5 & 0 & 5 \\
\hline $\begin{array}{l}\text { Realizaron actividades } \\
\text { de prevención de la } \\
\text { violencia de género }\end{array}$ & 5 & 0 & 5 \\
\hline $\begin{array}{l}\text { Realizaron cursos y/o } \\
\text { talleres con perspectiva } \\
\text { de género }\end{array}$ & 4 & 0 & 4 \\
\hline $\begin{array}{l}\text { Realizaron actividades } \\
\text { de sensibilización para la } \\
\text { igualdad de género }\end{array}$ & & & \\
\hline
\end{tabular}

Fuente: ONIGIES, 2017.

EI IPN, es una IES uniforme en las entidades con acciones para la igualdad, aunque tuvo solo tres acciones: una en Corresponsabilidad Familia, una en No Violencia y una en Sensibilización en Género. Fue la institución con mayor promedio de entidades con acciones (ver tabla 4).

Tabla 4

Entidades del IPN que realizan acciones de igualdad de género

\begin{tabular}{|l|c|c|c|}
\hline \multicolumn{1}{|c|}{ ACCIONES } & ACADÉMICAS & ADMINISTRATIVAS & TOTAL \\
\hline $\begin{array}{l}\text { Realizaron acciones } \\
\text { explícitas para promover } \\
\text { la corresponsabilidad }\end{array}$ & 54 & 36 & 90 \\
\hline $\begin{array}{l}\text { Realizaron actividades de } \\
\text { prevención de la violencia } \\
\text { de género }\end{array}$ & 54 & 36 & 90 \\
\hline $\begin{array}{l}\text { Realizaron actividades de } \\
\text { sensibilización para la } \\
\text { igualdad de género }\end{array}$ & 54 & 36 & 90 \\
\hline
\end{tabular}

Fuente: ONIGIES, 2017.

La UAS tuvo 10 acciones, con un promedio de 29 entidades, tanto académicas como administrativas, el promedio de las entidades académicas fue de 23.9 y el de entidades administrativas era de 5.66. Estuvieron repartidas en cinco ejes de la siguiente manera: una en Corresponsabilidad Familiar, una en Estadísticas y diagnósticos con Enfoque de Género, tres Legislación con Perspectiva de Género, dos en No Violencia y dos en

IQUAL. REVISTA DE GÉNERO E IGUALDAD, 2022, 5, 51-78

ISSN. 2603-851X

DOI. http://dx.doi.org/10.6018/iqual.483711 
Sensibilización en Género. La acción con mayor promedio de entidades tanto administrativas como académicas fue contar con mecanismos para la presentación de quejas por violencia de género (ver tabla 5 ).

Tabla 5

Entidades de la UAS que realizan acciones de igualdad de género

\begin{tabular}{|c|c|c|c|}
\hline ACCIONES & ACADÉMICAS & ADMINISTRATIVAS & TOTAL \\
\hline $\begin{array}{l}\text { Realizaron acciones } \\
\text { explícitas para promover } \\
\text { la corresponsabilidad }\end{array}$ & 10 & 1 & 11 \\
\hline $\begin{array}{l}\text { Publicaron informes de } \\
\text { labores con datos } \\
\text { desagregados por sexo. }\end{array}$ & 3 & 3 & 6 \\
\hline $\begin{array}{l}\text { Cuentan con diagnósticos } \\
\text { cuantitativos de igualdad } \\
\text { de género }\end{array}$ & 56 & 1 & 57 \\
\hline $\begin{array}{l}\text { Órganos internos para la } \\
\text { igualdad de género }\end{array}$ & 1 & 1 & 2 \\
\hline $\begin{array}{l}\text { Incluyen acciones a favor } \\
\text { de la igualdad de género } \\
\text { en los programas de } \\
\text { trabajo de sus titulares }\end{array}$ & 1 & 1 & 2 \\
\hline $\begin{array}{l}\text { Ejercieron recursos } \\
\text { económicos para la } \\
\text { igualdad de género }\end{array}$ & 1 & 1 & 2 \\
\hline $\begin{array}{l}\text { Cuentan con mecanismos } \\
\text { para la presentación de } \\
\text { quejas por violencia de } \\
\text { género }\end{array}$ & 44 & 41 & 85 \\
\hline $\begin{array}{l}\text { Realizaron actividades de } \\
\text { prevención de la violencia } \\
\text { de género }\end{array}$ & 41 & 1 & 42 \\
\hline $\begin{array}{l}\text { Realizaron cursos y/o } \\
\text { talleres con perspectiva } \\
\text { de género }\end{array}$ & 41 & 0 & 41 \\
\hline $\begin{array}{l}\text { Realizaron actividades de } \\
\text { sensibilización para la } \\
\text { igualdad de género }\end{array}$ & 41 & 1 & 42 \\
\hline
\end{tabular}

Fuente: ONIGIES, 2017. 
La BUAP tiene siete acciones en un promedio de 2.85 entidades, por tipo de entidad tiene un promedio de 1.28 administrativas y 1.83 administrativas con acciones para la igualdad de género. Las siete acciones están repartidas en los siguientes cinco ejes: una en Estadísticas y Diagnósticos con Enfoque de Género, una en Investigaciones y Estudios de Género, dos en Legislación con Perspectiva de Género, una en No Violencia y dos en Sensibilización en Género (ver tabla 6).

Tabla 6

Entidades de la BUAP que realizan acciones de igualdad de género

\begin{tabular}{|l|c|c|c|}
\hline \multicolumn{1}{|c|}{ ACCIONES } & ACADÉMICAS & ADMINISTATIVAS & TOTAL \\
\hline $\begin{array}{l}\text { Publicaron informes de } \\
\text { labores con datos } \\
\text { desagregados por sexo. }\end{array}$ & 1 & 2 & 3 \\
\hline $\begin{array}{l}\text { Cuentan con grupos } \\
\text { académicos con perspectiva } \\
\text { de género }\end{array}$ & 2 & 2 & 2 \\
\hline $\begin{array}{l}\text { Órganos internos para la } \\
\text { igualdad de género }\end{array}$ & 1 & 1 & 3 \\
\hline $\begin{array}{l}\text { Incluyen acciones a favor de } \\
\text { la igualdad de género en los } \\
\text { programas de trabajo de sus } \\
\text { titulares }\end{array}$ & 2 & 1 & 3 \\
\hline $\begin{array}{l}\text { Realizaron actividades de } \\
\text { prevención de la violencia de } \\
\text { género }\end{array}$ & 1 & 2 & 2 \\
\hline $\begin{array}{l}\text { Realizaron cursos y/o talleres } \\
\text { con perspectiva de género }\end{array}$ & 1 & 3 & 3 \\
\hline $\begin{array}{l}\text { Realizaron actividades de } \\
\text { sensibilización para la } \\
\text { igualdad de género }\end{array}$ & 1 & & \\
\hline
\end{tabular}

Fuente: ONIGIES, 2017.

La UV tenía nueve acciones en un promedio de 47.22 entidades administrativas y académicas. Por tipo de entidad el promedio era de 38.66 para las académicas y 8.55 para las administrativas. En cuanto a los ejes estaban repartidas en cinco de la siguiente manera: una en Corresponsabilidad Familiar, dos en Estadísticas y Diagnósticos con Enfoque de Género, dos Legislación con Perspectiva de Género, dos en No Violencia y dos en Sensibilización en Género. Cabe resaltar que la acción con la que más contaban las entidades de ambos tipos era contar con mecanismos para la presentación de quejas por violencia de género (ver tabla 7).

IQUAL. REVISTA DE GÉNERO E IGUALDAD, 2022, 5, 51-78

ISSN. 2603-851X

DOI. http://dx.doi.org/10.6018/iqual.483711 
Tabla 7

Entidades de la UV que realizan acciones de igualdad de género

\begin{tabular}{|c|c|c|c|}
\hline ACCIONES & ACADÉMICAS & ADMINISTRATIVAS & TOTAL \\
\hline $\begin{array}{l}\text { Realizaron acciones explícitas } \\
\text { para promover la } \\
\text { corresponsabilidad }\end{array}$ & 38 & 1 & 39 \\
\hline $\begin{array}{l}\text { Publicaron informes de } \\
\text { labores con datos } \\
\text { desagregados por sexo. }\end{array}$ & 13 & 3 & 16 \\
\hline $\begin{array}{l}\text { Cuentan con diagnósticos } \\
\text { cuantitativos de igualdad de } \\
\text { género }\end{array}$ & 7 & 1 & 8 \\
\hline $\begin{array}{l}\text { Incluyen acciones a favor de la } \\
\text { igualdad de género en los } \\
\text { programas de trabajo de sus } \\
\text { titulares }\end{array}$ & 43 & 3 & 46 \\
\hline $\begin{array}{l}\text { Ejercieron recursos } \\
\text { económicos para la igualdad } \\
\text { de género }\end{array}$ & 1 & 1 & 2 \\
\hline $\begin{array}{l}\text { Cuentan con mecanismos } \\
\text { para la presentación de quejas } \\
\text { por violencia de género }\end{array}$ & 154 & 61 & 215 \\
\hline $\begin{array}{l}\text { Realizaron actividades de } \\
\text { prevención de la violencia de } \\
\text { género }\end{array}$ & 42 & 2 & 44 \\
\hline $\begin{array}{l}\text { Realizaron cursos y/o talleres } \\
\text { con perspectiva de género }\end{array}$ & 4 & 3 & 7 \\
\hline $\begin{array}{l}\text { Realizaron actividades de } \\
\text { sensibilización para la } \\
\text { igualdad de género }\end{array}$ & 46 & 2 & 48 \\
\hline
\end{tabular}

Fuente: ONIGIES, 2017.

Por último, la UAEM tenía cuatro acciones en un promedio de 34.75 entidades totales, 31.75 en las académicas y 3 en las administrativas. Esas acciones estaban repartidas en dos ejes de la siguiente manera: dos en Legislación con Perspectiva de Género y dos en Sensibilización en Género (ver tabla 8).

Tabla 8

Entidades de la UAEM que realizan acciones de igualdad de género

\begin{tabular}{|l|c|c|c|}
\hline \multicolumn{1}{|c|}{ UAEM } & ACADÉMICAS & ADMINISTRATIVAS & TOTAL \\
\hline $\begin{array}{l}\text { Órganos internos para la } \\
\text { igualdad de género }\end{array}$ & 48 & 1 & 49 \\
\hline
\end{tabular}

IQUAL. REVISTA DE GÉNERO E IGUALDAD, 2022, 5, 51-78 


\begin{tabular}{|l|c|c|c|}
\hline $\begin{array}{l}\text { Incluyen acciones a favor } \\
\text { de la igualdad de género } \\
\text { en los programas de } \\
\text { trabajo de sus titulares }\end{array}$ & 48 & 9 & 57 \\
\hline $\begin{array}{l}\text { Realizaron cursos y/o } \\
\text { talleres con perspectiva de } \\
\text { género }\end{array}$ & 3 & 1 & 4 \\
\hline $\begin{array}{l}\text { Realizaron actividades de } \\
\text { sensibilización para la } \\
\text { igualdad de género }\end{array}$ & 28 & 1 & 29 \\
\hline
\end{tabular}

Fuente: ONIGIES, 2017.

\section{Conclusiones}

El incremento de las mujeres en las IES junto con la penetración de directrices internacionales generó la organización y manifestación de grupos feministas de estudiantes, académicas y personal administrativo para su defensa contra la violencia de género. La revisión de antecedentes muestra que primero hubo casos de violencia contra las mujeres, principalmente de hostigamiento y acoso sexual, después la reacción de la comunidad universitaria que se dio principalmente en la UNAM y después los cambios a nivel institucional.

Pero los antecedentes son enfáticos en que los cambios normativos requieren de cambios más profundos en la base. Es decir, en las conductas y prácticas cotidianas de las IES. Los casos recabados en los estudios que se revisaron mostraron cómo existe una ignorancia, incluso intencional, que provoca una resistencia a actuar contra casos de violencia sexual. Además, hubo casos que se dieron en relaciones jerárquicas de desigualdad, de profesores hacia alumnas. Por lo que la presión de la comunidad universitaria afectada hacia las autoridades ha mantenido su sentido al exigir un cambio normativo, cultural y epistemológico, como lo marca Palomar Verea.

Otro punto importante recabado de la revisión de antecedentes es la evidencia de procesos participativos en los reclamos contra casos de hostigamiento y acoso sexual. Los grupos organizados de feministas universitarias en palabras de Mingo ofrecen un ambiente de protección y empoderamiento a las víctimas dentro del cual se empoderan y apoyan en sus demandas de justicia. Por lo que se pugna por la creación de metodologías participativas en la creación de políticas con perspectiva de género.

Es así que las IES son, en términos de Weber, organizaciones conformadas por grupos de individuos que desempeñan distintos roles dentro de estructuras jerarquizadas de intereses. Estas organizaciones están imbuidas por rasgos de género inscritos en el cuerpo de las personas que conforman las estructuras, se imponen como discursos sobre IQUAL. REVISTA DE GÉNERO E IGUALDAD, 2022, 5, 51-78 ISSN. 2603-851X DOI. http://dx.doi.org/10.6018/iqual.483711 
sus cuerpos, y son de acuerdo a Butler performativos, es decir, se ejecutan cotidianamente. Por lo que los colectivos feministas al actuar de manera colectiva desde una posición de desigualdad y discriminación, como menciona Wolfesberger, se constituyen dentro de las IES como un clamor comunal democratizador.

Los resultados de los datos que se pueden descargar de ONIGIES mostraron evidencias interesantes de la participación de las mujeres dentro de los distintos roles institucionales. El más relevante es la proporción de mujeres dentro de la máximo autoridad colegiada de la Universidad Autónoma de Sinaloa que se corresponde con el alto índice dentro del Observatorio de Género ONIGIES; así como en los ejes institucionales y las acciones transversales. Mientras que por lo que respecta a la participación de las bases en la UNAM el colectivo La Red y sus movilizaciones se corresponden con los índices que presenta la institución.

En cuanto a la transversalización hay una correspondencia con la institucionalización; las IES que más indicadores de institucionalización tuvieron, contaron también con mayor participación de entidades académicas y administrativas en acciones de transversalización. La UNAM, UAS y UV son las IES que mejores acciones que desde lo legislativo hasta lo financiero presentan para dirimir y sancionar casos de violencia de género. Mientras que la UDG es la que peores indicadores presenta.

Por último, en este capítulo se revisaron las acciones institucionales contra las violencias de género desde la información que las propias IES reportan. Por lo que quedan pendiente conocer en las universidades como la UNAM, UAS, UV, BUAP, IPN, UAEM y UDG como se dirimen los casos específicos de violencia de género, así como el impacto de las campañas de conciliación y sensibilización.

\section{Bibliografía}

Barreto Ávila, M., \& Flores Garrido, N. (2016). Institucionalización, violencia de género y demandas desde la base. Nómadas (44), 201-218.

Briseño Maas, M. L., \& Bernabé Morales, T. E. (2019). Universidad pública y la atención a la violencia de género. Tequio, 5(2), 15-22.

Butler, J. (2002). Cuerpos que importan: sobre los límites materiales y discrusivos del sexo. Buenos Aires, Argentina: Paidos.

Cortazar Rodríguez, F. J. (2019). Acoso y hostigamiento de género de la Universidad de Guadalajara. Habla el estudiantado. Revista de estudios de género, La Ventana (50), 175-204.

IQUAL. REVISTA DE GÉNERO E IGUALDAD, 2022, 5, 51-78 
De Garay, A., \& del Valle-Diaz-Muñoz, G. (2011). Una mirada a la presencia de las mujeres en la educación superior en México. Revista Iberoamericana de Educación Superior, III (6), 3-30.

Lópes-Molina, S.-A., \& Vázquez-Guerrero, M. (2018). Las políticas de género en una universidad pública estatal:discrepancias institucionales. Revista Iberoamericana de Educación Superior, IX(25), 138-156.

Mercado, A., \& Planas, J. (2005). Evolución del nivel de estudios de la oferta de trabajo en México: una comparación con la Unión Europea. Revista mexicana de investigación educativa, 315-343.

Mingo, A., \& Moreno, H. (2015). Looking the other way: sexual harassment on campus. International Journal of Humanities and Social Sciences, 5 (9 (1)), 249-258.

Moreno, H., \& Mingo, A. (2020). Temor, desprecio y deseo como figuras del sexismo en la universidad. Nómadas, 51 , 13-29. doi: 10.30578/nomadas.n51a1.

ONIGIES. (31 de Agosto de 2020). Observatorio Nacional para la Igualdad de Género en las Intituciones de Educación Superior. Obtenido de Observatorio Nacional para la Igualdad de Género en las Intituciones de Educación Superior: https://observatorio.cieg.unam.mx/

Palomar Verea, C. (2005). La política de género en la educación superior. La Ventana(21), 7-43.

Varela Guinot, H. (2020). Las universidades frente a las violencias de género. El alcance limitado de los mecanismos formales. Revista Mexicana de Ciencias Políticas y Sociales(238), 49-80. doi: http://dx.doi.org/10.22201/fcpys.2448492xe.2020.238.68301.

Weber, M. (2004). Economía y Sociedad. México D.F., México: Fondo de Cultura Económica.

Wolfesberger, P. (2019). Lo político entre democracia y comunalidad. Revista Mexicana de Ciencias Políticias y Sociales, 64(237), 43-64. doi:http://dx.doi.org/10.22201/fcpys.2448492xe.2019.237.66390

Zamudio-Sánchez, F. J., Andrade-Barrera, M. A., Arana-Ovalle, R. I., \& Alvaro-Segura, A. A. (2017). Violencia de género sobre estudiantes universitarios(as). Convergencia Revista de Ciencias Sociales(75), 133-157.

IQUAL. REVISTA DE GÉNERO E IGUALDAD, 2022, 5, 51-78

ISSN. 2603-851X

DOI. http://dx.doi.org/10.6018/iqual.483711 
Cómo referenciar este artículo/How to reference this article:

Arce Casas, P.A. (2022). Políticas de género universitarias: Institucionalización, transversalización y participación de la comunidad. iQUAL. Revista de Género e Igualdad, 5, 51-78, doi: 10.6018/iqual.483711

Arce Casas, P.A. (2022). Políticas de género universitarias: Institucionalización, transversalización y participación de la comunidad. [University gernder policies: institutionalization, trasnversalization and community participation]. iQUAL. Revista de Género e lgualdad, 5, 51-78, doi: 10.6018/iqual.483711 